\title{
Use of Nail Dermoscopy in the Management of Melanonychia: Review
}

\author{
Michela Starace ${ }^{1}$, Aurora Alessandrini ${ }^{1}$, Nicolò Brandi ${ }^{1}$, Bianca Maria Piraccini ${ }^{1}$
}

1 Department of Experimental, Diagnostic and Specialty Medicine-Division of_Dermatology, University of Bologna, Bologna, Italy

Key words: pigmentation, melanonychia, dermoscopy, nail

Citation: Starace M, Alessandrini A, Brandi N, Piraccini BM. Review of the use of nail dermoscopy in the management of melanonychia: Review. Dermatol Pract Concept. 2019;9(1):38-43. DOI: https://doi.org/10.5826/dpc.0901a10

Published: January 31, 2019

Copyright: $@ 2019$ Starace et al. This is an open-access article distributed under the terms of the Creative Commons Attribution License, which permits unrestricted use, distribution, and reproduction in any medium, provided the original author and source are credited.

Funding: None.

Competing interests: The authors have no conflicts of interest to disclose.

Authorship: All authors have contributed significantly to this publication.

Corresponding author: Michela Starace MD, PhD, Department of Experimental, Diagnostic and Specialty Medicine-Division of Dermatology, University of Bologna, Bologna, Italy, Via Massarenti, 1, 40138 Bologna, Italy. Email: michela.starace2@unibo.it

ABSTRACT The term melanonychia describes a black-brown-gray pigmentation of the nail plate that, in most cases, appears as a longitudinal band. Melanonychia can be observed at any age, affecting one digit or several digits, in both fingernails and toenails. Its causes can be difficult to differentiate clinically and depend on the number of bands and on their color, edge, and width. For this reason, especially in adults and when the pigmentation is localized in one single digit, biopsy and histopathological examination still represent the gold standard for a definitive diagnosis. Dermoscopy should be used routinely in the evaluation of a pigmented nail, as it provides important information for the management of melanonychia and can help avoid unnecessary nail biopsies. In cases of melanonychia it is important to establish whether the pigment is melanin or not and to determine whether the pigmentation of melanin is due to activation or proliferation and whether it is benign or malignant.

\section{Introduction}

The term melanonychia describes a black-brown-gray pigmentation of the nail plate. Although nail matrix melanocytes are normally quiescent, these cells can eventually become activated with or without proliferation, thus inducing the pigmentation of the nail plate. Melanonychia can present itself in many different ways, but most frequently it appears as a longitudinal band (longitudinal melanonychia) that starts from the proximal margin of the nail and extends to the distal margin, following the growth of the nail. Less commonly, the pigment can be transverse (transverse melanonychia) [1] or even involve all of the nail plate (total melanonychia). Melanonychia can be observed at any age, affecting one digit or several digits, in both fingernails and toenails.

The causes of longitudinal melanonychia can be difficult to differentiate clinically and, first of all, depend on the number of the bands and on their color, edge, and width. Biopsy and histopathological examination is recommended when a longitudinal melanonychia occurs in an adult and it is localized in one single digit, even more so in the absence of local or systemic causes that may explain its onset. The real problem of how to distinguish whether the lesion is benign or malignant remains, as an early diagnosis of melanoma can be crucial for the prognosis. 
With this in mind, dermoscopy should be used routinely in the evaluation of a pigmented nail, as it provides important information for the management of melanonychia and can help to avoid unnecessary nail biopsies. In case of melanonychia, it is important to follow 3 steps: (1) to establish whether the pigment is melanin or not, (2) to determine whether the pigmentation of melanin is due to activation or proliferation, and (3) if it is a proliferation, to determine whether it is benign or malignant [2].

\section{Discussion}

When performing dermoscopy on nail pigmentation, it is important to observe all the visible part of the nail unit where it is possible to put the dermatoscope. Moreover, it is fundamental to remember that the use of gel is recommended for the examination of any pigmented lesions because it increases the visibility through the nail plate, even if it is more difficult with the gel because of the tendency to slide off the plate.

First of all, dermoscopy permits differentiating melanocytic from nonmelanocytic pigmentation, particularly the quite common brown-black nail discolorations due to subungual hematoma or fungal infection [3]. In fact, melanocytic pigmentation is generally brown-black, within the nail plate and with a longitudinal aspect, whereas exogenous pigmentation typically includes the spectrum of color of the substance that adheres to the nail plate and does not always have a longitudinal appearance located near the nail folds or in the center of the nail plate. The most common causes of nonmelanocytic pigmentation include subungual hematoma, fungal melanonychia, and green nails. Subungual hematoma is one of the most frequent causes of brown-black nail pigmentation, and blood can be recognized by the presence of the red-purple-brown globule pattern within the nail plate and the homogeneous color of the pigmenta-

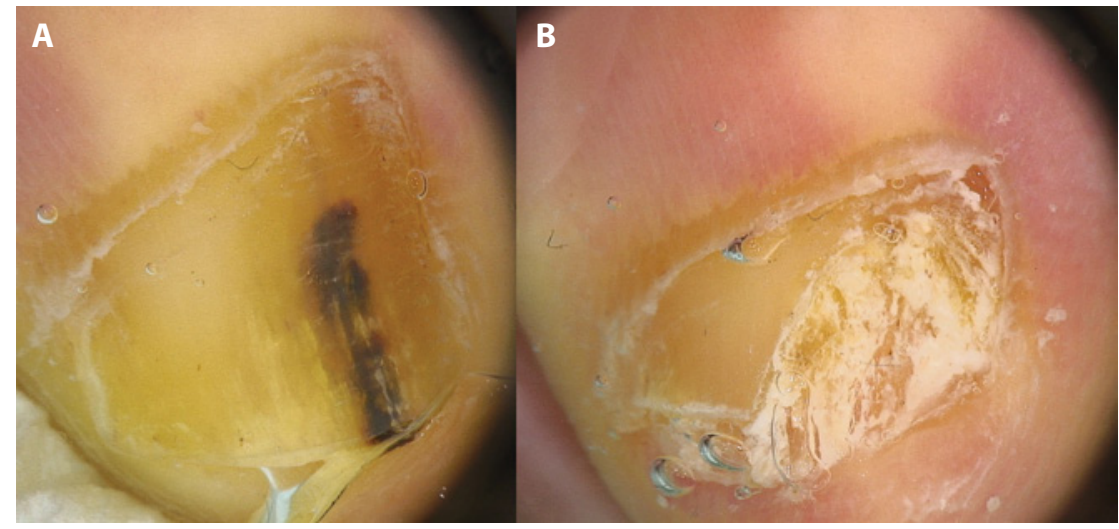

Figure 1. Dermoscopy of a brown-black nail pigmentation due to subungual hematoma. Red-purple globule pattern within the nail plate with homogeneous color of the pigmentation peripherally fading (A). The pigmentation disappears with nail clipping (B). [Copyright: (C)2019 Starace et al.]

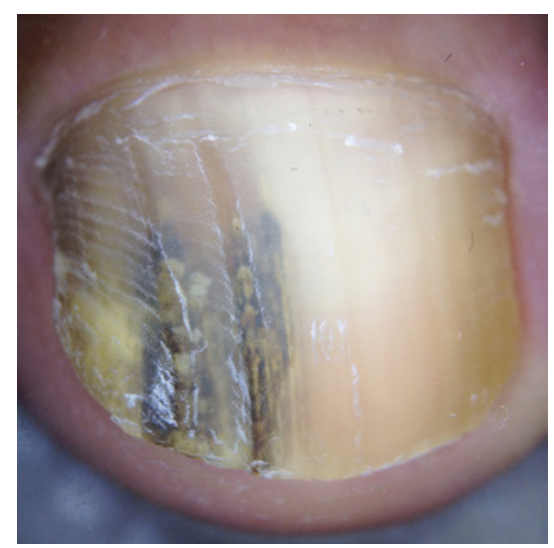

Figure 2. Melanonychia due to onychomycosis caused by Trichophyton rubrum variant melanoid. [Copyright: (C2019 Starace et al.]

tion, which peripherally fades and lacks the longitudinal lines of true melanonychia (Figure 1). Fungal melanonychia is a rare variant of onychomycosis caused by Trichophyton rubrum variant melanoid, which can present itself with a longitudinal band on the nail plate, thus further complicating differential diagnosis. Dermoscopy of fungal melanonychia reveals a usually brown band of melanonychia, with thick subungual hyperkeratosis and yellow and brown scales, wider at the distal ends and focalizing on reddish hue that may be related to traumatic hemorrhages from subungual hyperkeratosis; moreover, there are no visible inclusions of melanin, supporting a nonmelanocytic origin of the pigmentation [4] (Figure 2).

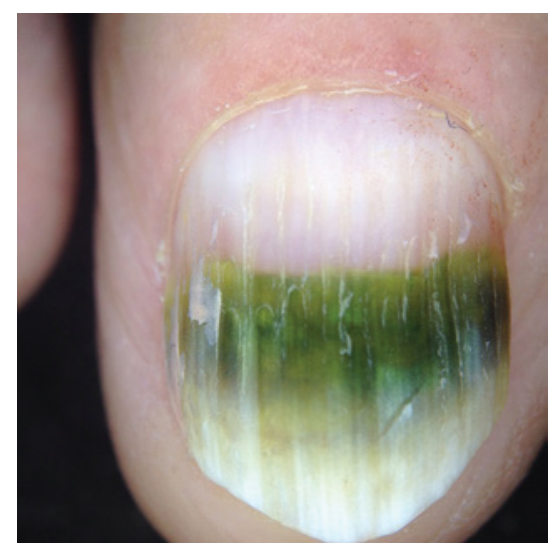

Figure 3. Dermoscopy of green nails due to Pseudomonas aeruginosa colonization of the nail. The convexity of the onycholytic border is linear. [Copyright: (C2019 Starace et al.]

In green nails, the pigmentation is due to bacterial colonization by Pseudomonas aeruginosa and is characterized by a bright green color that fades to yellow, laying under the nail plate [5] (Figure 3).

If the pigmentation is due to melanin produced by nail matrix, the second step of melanonychia management is to identify whether the pigmentation is due to an activation or proliferation. The number of digits involved is an important diagnostic clue: if more than one digit is affected, it is likely to be a melanocytic activation. The dermoscopic pattern that suggests a melanocytic activation is a gray background of the band with thin grayish regular and parallel lines (Figure 4); moreover, hyperpigmentation of the nail can also be associated with 


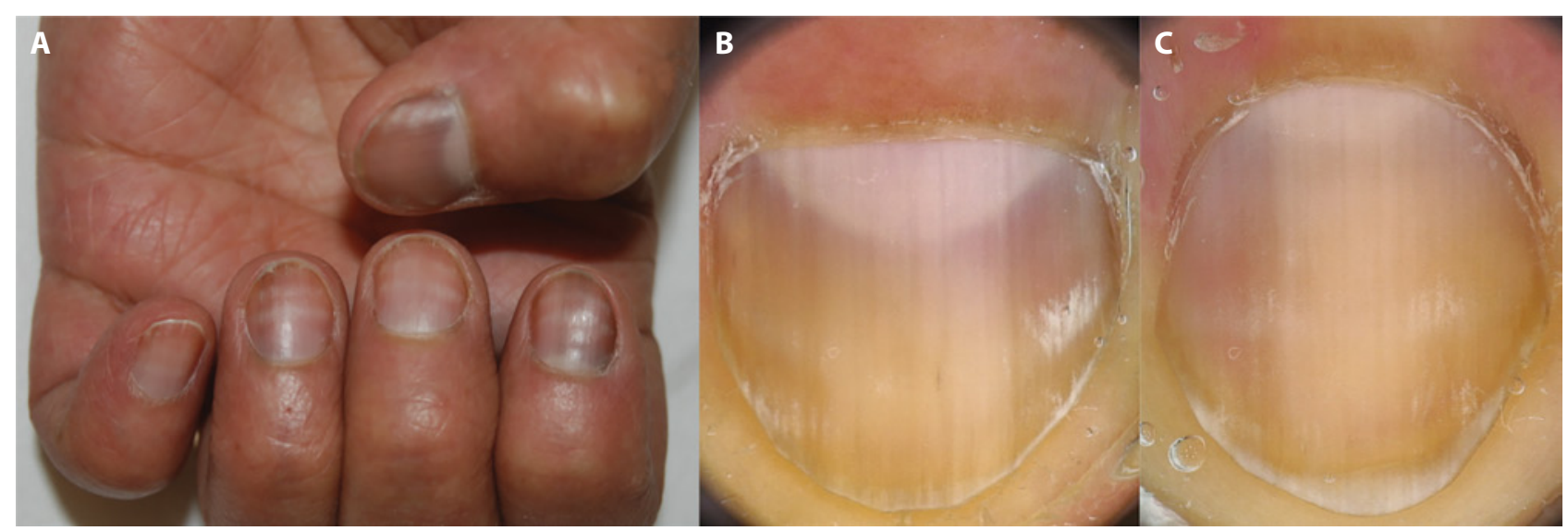

Figure 4. Clinical picture of melanocytic activation affecting all fingernails during combined chemotherapy (A); onychoscopy reveals gray background of the bands with thin grayish regular and parallel lines, associated with Muehrcke lines, which appear as white parallel transverse bands (B, C). [Copyright: @2019 Starace et al.]

a hyperpigmentation of the periungual skin [6]. When multiple nails present longitudinal melanonychia, it is important to consider a normal ethnic/racial variant, particularly in darker skin phototypes; the use of drugs; mechanical factors such as frictional melanonychia and onychotillomania; pregnancy; or an inflammatory disease or the manifestation of one of the multiple signs of a syndrome, such as Peutz-Jeghers syndrome and Laugier-Hunziker syndrome [5]. Frictional melanonychia is a very common form of melanocytic activation and is typically localized only on the fifth and/or fourth toenails, due to the frequently chronic friction of these digits with the shoes. The band is usually grayblack and its background is brown with parallel thin lines (Figure 5); in some cases, the friction of the toenails could induce trauma in the capillaries, thus leading to the appearance of red spots or splinter hemorrhages that reinforce the traumatic etiology of the pigmented band. In a similar way, in onychotillomania the mechanical trauma of biting of the proximal nail folds induces an activation of the melanocytes. In these cases, however, it is frequent to observe more digits involved and signs of chewing such as crusts or wounds on the most affected digits.

Dermoscopy of melanocytic nail pigmentation is difficult to interpret, as there are still not uniform criteria that allow us to differentiate melanonychia due to benign melanocytic proliferation, ie, nail matrix lentigo or nevus, from melanonychia due to malignant melanocytic proliferation [7].

The first cutoff in cases of longitudinal melanonychia due to proliferation is the age of the patient since nail melanoma is rare in children; only 20 cases are described in the literature, frequently in darker skin phototypes [6-10]. Dermoscopic patterns that suggest melanoma in children are rapid evolution of brown background with longitudinal brown to black lines with irregular degree of color pigmentation, spacing or varying thickness and ending abruptly or having a parallel distribution. However, these features can also be seen in nail matrix nevi in children and thus their specificity is low. For this reason, dermoscopy could have less value in childhood melanonychia [11].

Nonetheless nail matrix nevi may be present at birth; they can also occur with age. Nail matrix nevi affect fingernails more frequently than toenails, most often the thumb. Usually, the nail has one or more longitudinal heavily pigmented bands whose size can vary from a few millimeters to the whole nail and whose color may not be homogeneous and more or less dark [3]. Dermoscopic patterns that suggest a nail matrix nevus are the presence of a brown background with longitudinal brown to black regu-

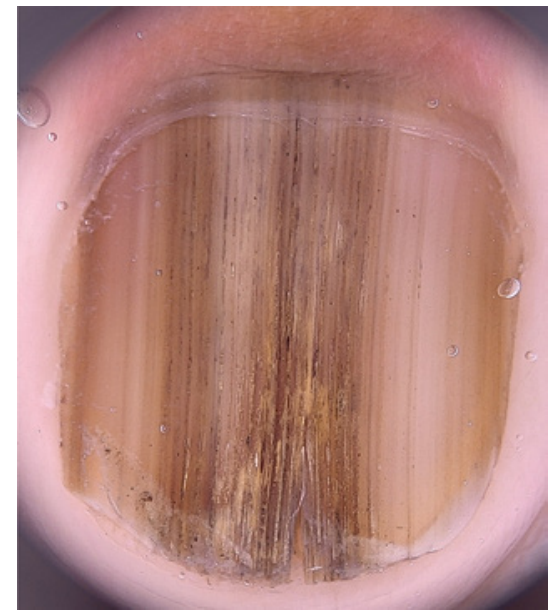

Figure 5. Nail dermoscopy of frictional melanonychia of the fifth toenail. Nail pigmentation is gray-brown with a brown background with parallel thin lines. [Copyright: (C2019 Starace et al.]

lar and parallel lines with regular spacing and thickness. A new dermoscopic sign that can indicate a regression of a nevus and not represent a warning sign of a melanoma, especially in children, is the presence of black dots along melanocytic lines [12]. These dots are black, with a regular size and shape (less than $0.1 \mathrm{~mm}$ ), and are distributed along the pigmented lines; sometimes they form a shallow pit at the periphery while other times it is possible to find them within the pigmented lines, often interrupting them. The authors have explained the presence of these dots as an accumulation of melanin derived from a cluster of nevus cells that migrate upward 


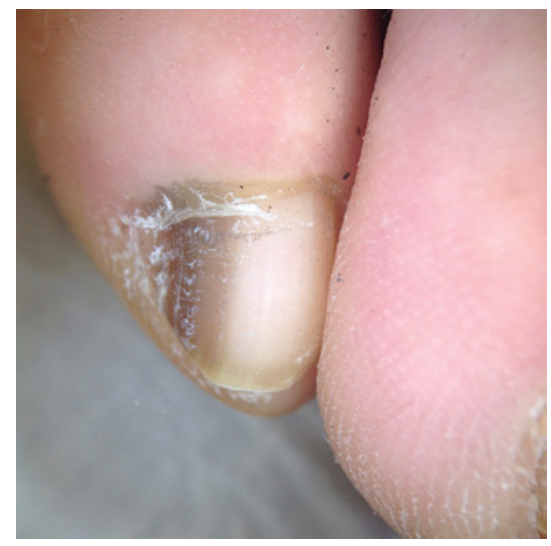

Figure 6. Dermoscopy of a nail matrix nevus in a 5-year-old child. Brown background with longitudinal brown to black irregular and not parallel lines with irregular spacing and thickness, with the presence of black dots along melanocytic lines. The pseudo-Hutchinson sign is also evident in the proximal nail fold. [Copyright: @2019 Starace et al.]

from the dermo-epidermal junction. In most young patients, the disappearance of the dots will occur over time and will accompany the fading of melanonychia. Dark bands are associated with pseudo-Hutchinson sign, because the dark nail plate pigmentation is visible through the transparent nail fold (illusory phenomenon); the pigmentation may be homogeneously distributed or darker bands may appear over diffuse pale pigmentation (Figure 6). Periungual pigmentation is typical in congenital nevi and may involve both the nail proximal fold and the hyponychium, thus alarming the clinician. In children, it is not uncommon to notice a gradual darkening and enlargement of the band that may have a proximal part broader than the distal (triangular shape); thinning and fissuring of the pigmented nail plate may also occur. It is also quite common to observe a gradual fading of the band with age [13]. This "regressing nevoid nail melanoma in childhood" is unique to children and not indicative of regression of the nevus but may just indicate decreased melanocytic activity from nevus cells [14].

The diagnostic accuracy of longitudinal melanonychia has improved over

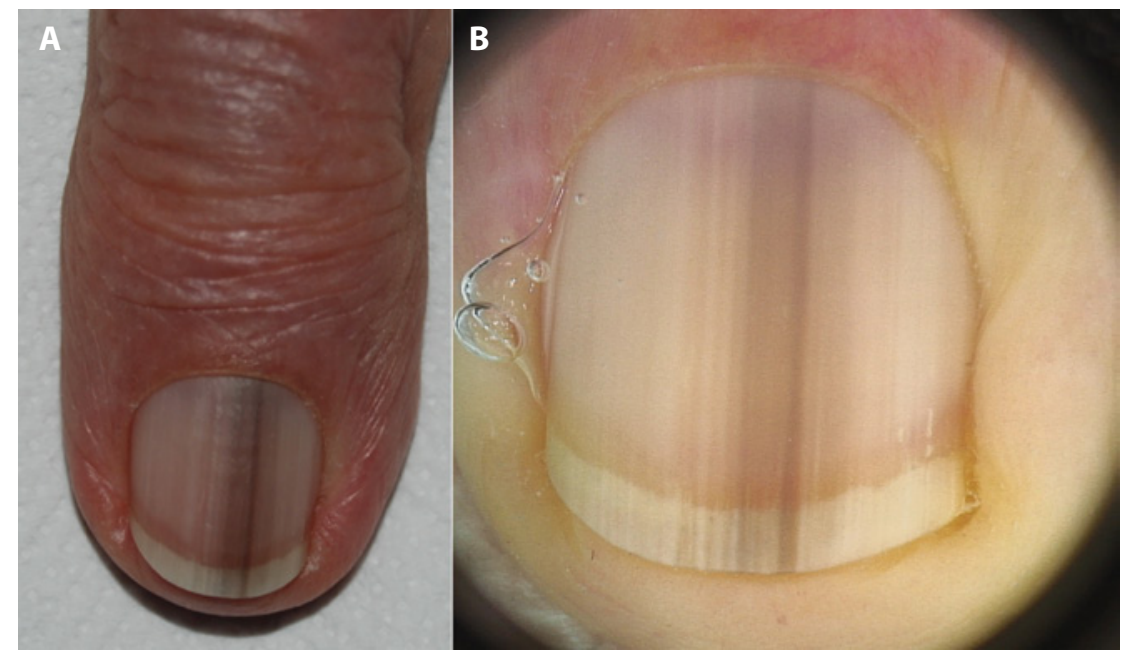

Figure 7. Clinical picture of nail melanoma of the II digit of right hand (A); a brown-black band of melanonychia characterized by irregular lines in their thickness, spacing, color, and parallelism at dermoscopy (B). [Copyright: (O2019 Starace et al.]

the years, first for the use of the ABCDs rule for nail pigmentation [15] and second for the introduction of nail dermoscopy [16] and its criteria for benign and malignant lesions [17]. In fact, even if the ABDEF rule is a useful clinical tool to suspect a melanoma, nail dermoscopy offers further help and permits seeing with high magnification the details of the pigmentation aspects not visible to the naked eye, enabling the visualization of different signs of melanoma. However, it is important to remember that dermoscopy is not always reliable in the evaluation and management of the pigmented nail [18], since there are some conditions for which dermoscopy is not performable, such as thickened nails, blurred borders of the lesions, and a nail plate that is totally black $[5,18]$. Because of its rarity, there are only a few studies focused on melanoma dermoscopic features; therefore, dermoscopy should still be assisted by the clinical features and the history of the nail pigmentation.

When only one digit is involved by melanonychia, a proliferative process has to be considered and a diagnosis of melanoma suspected. The clinical features of the band can be very different: the color can be more or less pronounced and homogeneous, the borders can be well defined or less sharp, the width can range from $1 \mathrm{~mm}$ to spanning the entire nail plate, the corresponding nail plate can show some changes or be completely normal, and, finally, brown-black periungual pigmentation (Hutchinson sign) may or may not be present.

The dermoscopic features of benign melanocytic proliferation are characterized by gray or brown homogeneous color of the background and brown bands with multiple thin brown lines; these lines are usually regular and parallel to each other, with regular spacing and thickness and similar shades of brown throughout the whole lesion, as in nevi. On the contrary, dermoscopic features suggestive of nail melanoma include a brown to black background of the band with longitudinal lines irregular in their thickness, spacing, color, or parallelism [17] (Figure 7). However, this rule is not always reliable, as it is possible to find lines that are irregular in width or color also in benign lesions $[5,18]$.

A recent study identified 3 important dermoscopic features that could help to distinguish whether the band is benign or malignant. In particular, a band involving more than two-thirds of the nail plate, a color gray to black, and the presence of nail dystrophy increase 3 times the risk of detecting a nail melanoma [19]. 


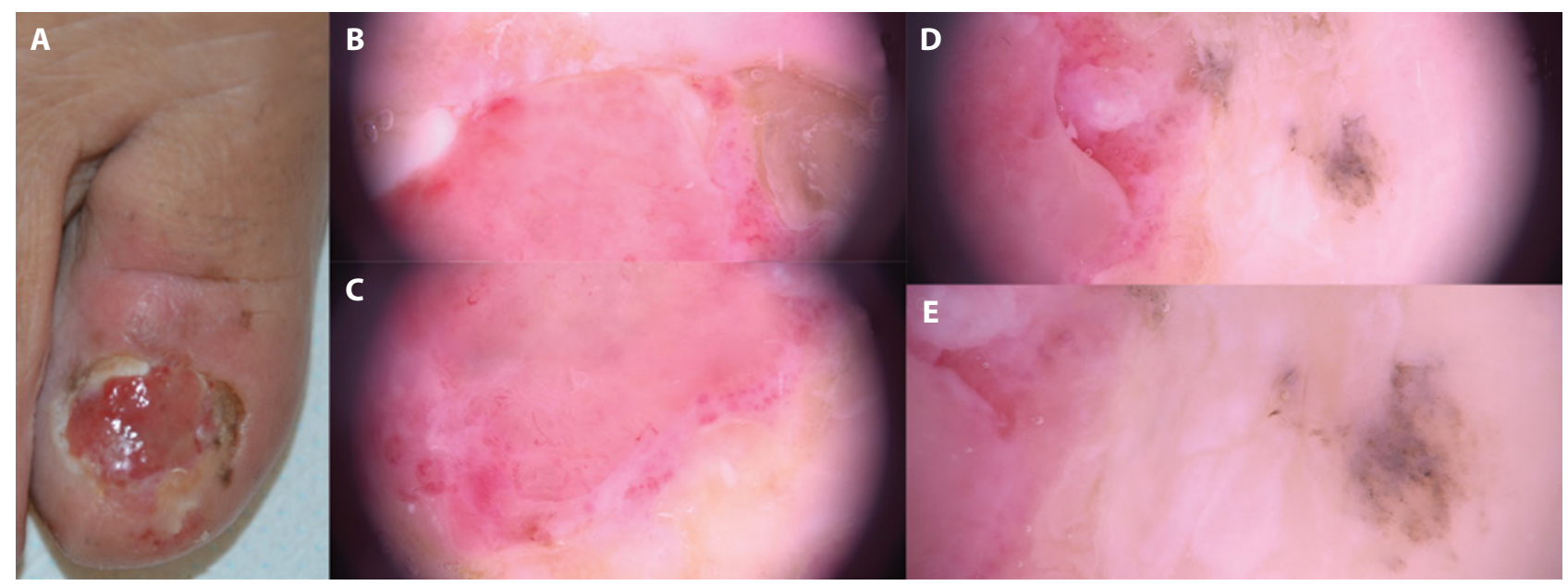

Figure 8. Amelanotic melanoma of the toenail. At clinical examination the absence of the nail plate with ulceration is evident (A); dermoscopy magnifies a nail bed nodule with irregular vascular pattern with milky red veil and Hutchinson sign at hyponychium (B-E). [Copyright: (C)2019 Starace et al.]

Dermoscopy of the free edge of the distal nail plate can also be very helpful in distinguishing whether the pigment is produced by proximal or distal nail matrix. In fact, if the pigment is in the dorsal part of the free edge its origin comes from the proximal nail matrix, while if the pigment is in the ventral part of the free edge its origin is the distal nail matrix [5]. Moreover, dermoscopic features of the nail plate and periungual tissues also offer interesting information for the selection of cases in which pathological examination is indicated, permitting the evaluation of Hutchinson sign, one of the most important signs of malignancy. Dermoscopically, this sign appears as a brown-black periungual pigmentation, characterized by polychromia and asymmetry. However, the most important role of nail dermoscopy is the ability to detect the micro-Hutchinson sign, a periungual pigmentation visible only with a dermatoscope and not with the naked eye, which represents an initial sign of radial growth into adjacent tissue. Moreover, the micro-Hutchinson sign could be present also in amelanotic melanoma, a malignant lesion characterized by the lack of melanin pigment. The clinical features of this type of melanoma are the absence of the nail plate and a nail dystrophy due to nail bed hyperkeratosis or ulceration; other dermoscopic patterns typical of amelanotic melanoma are eroded nail bed nodule, vascular pattern characterized by a red discoloration with a milky-red veil and variable aspect of vessels, with some appearing as dots and others as irregular lines. The color of amelanotic melanoma is white in the center of the lesion and red in the periphery (Figure 8) and can also correlate with the duration of the lesion. In older lesions the predominant color is dark red, probably due to the presence of crusts and vascular disarrangement, with irregular vessels assuming a mix of hairpin, linear, or dotty aspect; on the other hand, younger lesions have a lighter red color and present prominently dotted vessels [20]. Distinguishing this tumor from pyogenic granuloma could be difficult sometimes.

In summary, dermoscopy is useful for the diagnosis of melanoma in adults especially in 2 cases: when there is the micro-Hutchinson sign, ie, when melanoma is present as an ulcerated nodule of the nail bed, and when melanoma is present as a band of longitudinal melanonychia, not associated with periungual pigmentation and/or nail plate changes. A useful algorithm may help us to understand what is the best management in case of suspected nail apparatus melanoma and possibly allow an earlier diagnosis with detection of in situ nail melanoma [21].

False-negative and false-positive dermoscopic results are not rare, especially in cases of benign lesions with irregular lines. Nevertheless, a delayed diagnosis of melanoma remains common and a poor prognosis is often attributable to longstanding disease. The misdiagnosis rate has been found to be higher than $20 \%$ [22].

Intraoperative dermoscopy enables visualization of the origin of the pigmentation, revealing aspects not observed when the nail plate is interposed between the pigmented lesion and the dermatoscope [23]. This type of dermoscopy is more reliable and accurate than plate dermoscopy and has high sensitivity and specificity in differentiating pigmented nail lesions, albeit is a part of an invasive procedure and cannot be performed in all cases of melanonychia. With the intraoperative method, 4 dermoscopic features have been recognized: regular gray pattern, typical of hypermelanosis; regular brown pattern, typical of benign melanocytic hyperplasia; regular brown pattern with globules or blotches, typical of melanocytic nevi; and irregular pattern, typical of melanoma. The use of intraoperative procedure may facilitate selecting the best site to perform biopsy [24]. 
There are no evidence-based studies that determine the ideal frequency of dermoscopic follow-up in patients with longitudinal melanonychia nor any that make precise dermoscopic criteria to decide when the pigmented lesions require biopsy [3]. One suggestion is to monitor the patient every 6 months with dermoscopy in cases of benign melanocytic lesions and to have a strict follow-up every 3 months in cases of irregular pigmentation without other signs of malignancy [25]. Nevertheless, the gold standard for a definitive diagnosis of nail pigmentation still remains histopathology [26,27].

\section{Conclusions}

Dermoscopy of the nail (onychoscopy) is nowadays used routinely, as it provides important information, giving specific criteria for the dermoscopic assessment of nail pigmentation.

The causes of melanonychia vary from subungual hematoma, to a fungal and/or bacterial infection, to a melanocytic lesion (nevus melanoma, etc), and the differential diagnosis is not easy for the nonexpert dermatologist. In addition, the management is often a reason for concern. Dermoscopy helps to identify the type of pigment and to determine the origin of the pigment. It is an important technique for the evaluation of melanocytic pigmentation; however, it has to be added to clinical data and, in cases of suspected melanoma, to biopsy for pathology.

\section{References}

1. Mirfazaelian H, Alaeen A, Daneshbod Y. Transverse melanonychia. Br J Hosp Med (Lond). 2014;75(7):413.

2. Lencastre A, Lamas A, Sà D, et al. Onychoscopy. Clin Dermatol. 2013;31(5):587-593.

3. Tosti A, Piraccini BM, de Farias DC. Dealing with melanonychia. Semin Cutan Med Surg. 2009;28(1):49-54.

4. Wang YJ, Sun PL. Fungal melanonychia caused by Trichophyton rubrum and the value of dermoscopy. Cutis. 2014;94(3):E5-E6.

5. Piraccini BM, Dika E, Fanti PA. Tips for diagnosis and treatment of nail pigmentation with practical algorithm. Dermatol Clin. 2015;33(2):185-195.

6. Braun R, Baran R, Saurat JH, Thomas L. Surgical pearl: dermoscopy of the free edge of the nail to determine the level of nail plate pigmentation and the location of its probable origin in the proximal or distal nail matrix. J Am Acad Dermatol. 2006;55(3):512513.

7. Goettmann-Bonvallott S, André J, Belaich S. Longitudinal melanonychia in children: a clinical and histopathologic study of 40 cases. J Am Acad Dermatol. 1999;41(1):17-22.

8. Lyall D. Malignant melanoma in infancy. J Am Med Assoc. 1967;202(13):1153.
9. Uchiyama M, Minemura K. Two cases of malignant melanoma in young persons. Nippon Hifuka Gakkai Zasshi. 1979;89:668.

10. Iorizzo M, Tosti A, Di Chiacchio N, et al. Nail melanoma in children: differential diagnosis and management. Dermatol Surg. 2008;34(7):974-978.

11. Tosti A, Piraccini BM, Cagalli A, Haneke E. In situ melanoma of the nail unit in children: report of two cases in fair-skinned Caucasian children. Pediatr Dermatol. 2012;29(1):79-83.

12. Murata Y, Kumano K. Dots and lines: a dermoscopic sign of regression of longitudinal melanonychia in children. Cutis. 2012;90:293-296.

13. Tosti A, Baran R, Morelli R, et al. Progressive fading of a longitudinal melanonychia due to a nail matrix melanocytic nevus in a child. Arch Dermatol. 1994;130(8):1076-1077.

14. Kikuchi I, Inoue S, Sakaguchi E, Ono T. Regressing nevoid nail melanosis in childhood. Dermatology. 1993;186:88-93.

15. Levit EK, Kagen MH, Scher RK, Grossman M, Altman E. The $\mathrm{ABC}$ rule for clinical detection of subungual melanoma. J Am Acad Dermatol. 2000;42(2):269-274.

16. Ronger S, Touzet S, Ligeron C, et al. Dermoscopic examination of nail pigmentation. Arch Dermatol. 2002;138(10):1327-1333.

17. Thomas L, Dalle S. Dermoscopy provides useful information for the management of melanonychia striata. Dermatol Ther. 2007;20(1):3-10.

18. Di Chiacchio N, Hirata AH, Daniel R, et al. Consensus on melanonychia nail plate dermoscopy. An Bras Dermatol. 2013;88(2):309-313.

19. Benati E, Riberio S, Longo C, et al. Clinical and dermoscopic clues to differentiate pigmented nail bands: an International Dermoscopy Society study. J Eur Acad Dermatol Venereol. 2017;31(4):732-736.

20. Phan A, Dalle S, Touzet S, Ronger-Savlé S. Balme B, Thomas L. Dermoscopic features of acral lentiginous melanoma in a large series of 110 cases in a white population. Br J Dermatol. 2010;162(4):765-771.

21. Starace M, Dika E, Fanti PA, et al. Nail apparatus melanoma: dermoscopic and histopathologic correlations on a series of 23 patients from a single centre. J Eur Acad Dermatol Venereol. 2018;32(1):164-173.

22. Phan A, Touzet S, Dalle S, et al. Acral lentiginous melanoma: a clinicoprognostic study of 126 cases. Br J Dermatol. 2006;155(3):561-569.

23. Hirata SH, Yamada S, Enokihara MY, et al. Patterns of nail matrix and bed of longitudinal melanonychia by intraoperative dermatoscopy. J Am Acad Dermatol. 2011;65(2):297-303.

24. Hirata SH, Yamada S, Almeida FA, et al. Dermoscopic examination of the nail bed and matrix. Int J Dermatol. 2006;45(1):28-30.

25. Sawada M, Yokota K, Matsumoto T, et al. Proposed classification of longitudinal melanonychia based on clinical and dermoscopic criteria. Int J Dermatol. 2014;53(5):581-585.

26. Ackerman AB. Malignant melanoma in situ: the flat, curable stage of malignant melanoma. Pathology. 1985;17(2):298-300.

27. Ruben BS. Pigmented lesions of the nail unit: clinical and histopathologic features. Semin Cutan Med Surg. 2010;29(3):148-158. 